\title{
Reflexiones sobre la presencia de regularidades en los fenómenos sociales y sobre la posibilidad de investigarlas desde un monismo metodológico ${ }^{1}$
}

\author{
Héctor Salas Harms*
}

\begin{abstract}
Resumen
La historia de la ciencia perfila un orden temporal en el que la evolución de las disciplinas y aun la de sus especialidades ha culminado en su maduración científica; también perfila un orden en el cual sus teorías han alcanzado precisión al explicar y predecir sus fenómenos. Tal orden corresponde a la complejidad de los eventos que estudian esos campos del conocimiento; actualmente, madurez y precisión son característicos solamente en las ciencias naturales
\end{abstract}

Las disciplinas sociales están hoy aún en una etapa pre-paradigmática, pre-científica, con disensión en sus comunidades de investigación aun sobre la índole de los hechos sociales: el dualismo metodológico piensa que en la naturaleza hay uniformidad y leyes universales, pero que los eventos humanos deben sólo comprenderse e interpretarse casuísticamente; por su parte, el monismo metodológico busca discernir y explicar regularidades mediante pruebas de hipótesis, modelos causales, rigor metodológico y resultados generalizables y replicables.

Este texto presenta algunos hechos y regularidades en administración y en economía que pueden establecerse como ciertos y válidos para todo investigador. Asimismo, se muestra cómo pueden definirse mediante análisis ex ante los determinantes de diversos fenómenos sociales, inclusive de aquéllos cuyas variables no son susceptibles de ser cuantificadas.

\footnotetext{
${ }^{1}$ Partes de este texto aparecen con el título "Los hechos y las regularidades en los fenómenos sociales", en Memoria del XI Foro de Investigación: Congreso Internacional de Contaduría, Administración e Informática, FCA-ANFECA, UNAM, México, 2006, pp. 1-12.

* Investigador de la División de Investigación de la Facultad de Contaduría y Administración, unam. Correo electrónico: hsalas@correo.fca.unam.mx
} 
Héctor Salas Harms

Palabras clave: filosofía de las disciplinas sociales, monismo metodológico, regularidades, hechos objetivos, dualismo metodológico.

Fecha de recepción: 04-12-2006 Fecha de aceptación 05- 07-2007

\title{
Some Reflections on the Presence of Regularities in the Social Phenomena, and the Possibility of Studying them According to a Methodological Monism
}

\begin{abstract}
History of science outlines a temporary order in which the evolution of the disciplines and even that of their specialties has culminated in their scientific maturity, as well as an order in which their theories have attained precision in explaining and predicting their phenomena. This order can be seen to correspond to the complexity of the events which are studied by each field, and at present both maturity and precision are distinctive in the natural sciences only.
\end{abstract}

The social disciplines remain today in a pre-paradigmatic, pre-scientific stage of development -research communities disagreeing even on the nature of social facts. Methodological dualism claims that the natural world exhibits uniformity and universal laws, but human events can at best be comprehended and interpreted on a case by case basis; while methodological monism attempts to discern and explain regularities through the testing of hypotheses with causal models, methodological thoroughness and findings that can be generalized and replicated.

Accordingly, in line with the second point of view, facts and regularities in management and economics which can be established with certainty and validity for all are discussed in this paper. And it is also shown how the determinants of different social phenomena can be defined by analyses ex ante, including those whose variables are not susceptible of quantification.

Keywords: philosophy of social science, methodological monism, regularities, objective facts, methodological dualism.

\section{Introducción}

Cl alto nivel de complejidad inherente a los eventos de la naturaleza humana, en

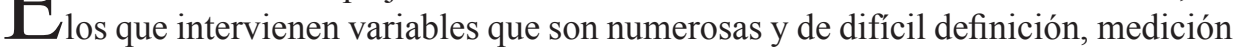
y observación, y que muestran relaciones causales marcadamente probabilísticas 
Reflexiones sobre la presencia de regularidades en los fenómenos sociales

y sobre la posibilidad de investigarlas desde un monismo metodológico

(frente a una causalidad que evoca esquemas determinísticos en muchos de los fenómenos de la naturaleza que se encuentran directamente al alcance de los sentidos), ha dividido a las comunidades de investigación de las disciplinas sociales con respecto a los objetivos, criterios de validez y metodologías, entre otros aspectos, que deben caracterizar al estudio de los hechos sociales, concurriendo en ellas actualmente un dualismo y un monismo metodológicos. ${ }^{2}$

La primera de estas posturas considera viable únicamente la descripción, comprensión e interpretación casuística y no generalizable de esos fenómenos, y los estudia desde métodos que conducen a resultados de subjetividad inherente, sosteniendo implícita o explícitamente que sólo en la naturaleza pueden encontrarse regularidades y establecerse leyes; mientras que la segunda, llevando a cabo su investigación en las áreas cuantitativas de las disciplinas sociales, se aboca a discernir y explicar regularidades en los hechos humanos desde modelos causales para prueba de hipótesis con rigor metodológico, produciendo conclusiones generalizables y replicables por otros investigadores. Este grupo, insistiendo en renunciar a la subjetividad, enfoca su trabajo particularmente a áreas en las cuales se han desarrollado elaboradas metodologías convencionales para definir y cuantificar las variables de índole no empírica propias de los eventos sociales, con la finalidad de generar resultados e implicaciones para la teoría que se presenten como válidos para todo investigador.

El propósito de este trabajo es mostrar la presencia de regularidades en los fenómenos de la naturaleza humana, en las que se asocian necesariamente sus causas y efectos, así como la posibilidad de establecer tales regularidades y otros hechos sociales como hechos objetivos y conocimiento cierto y válido para todos mediante proyectos replicables. Asimismo, y en paralelo a la aplicación de metodologías de corte naturalista $^{3}$ en las disciplinas sociales, se analizan los criterios naturalistas de cientificidad tanto para campos del conocimiento como para teorías, a modo de referencia para la investigación en dichas disciplinas.

\footnotetext{
${ }^{2}$ Igualmente, en filosofía de la ciencia hay dos posturas sobre los criterios aplicables al estudio de los hechos sociales. El naturalismo o monismo metodológico aplica normativamente también en las disciplinas sociales los modelos de la filosofía de las ciencias naturales; mientras que el dualismo metodológico afirma que la investigación de los fenómenos sociales debe orientarse y sus resultados deben justificarse desde modelos de cientificidad y racionalidad alternativos y específicos para los mismos. (Existe en filosofía de la ciencia otro sentido para el término 'naturalismo', desde cuya tesis las teorías filosóficas de las ciencias deben contrastarse empíricamente con la historia real de las ciencias.) (Cf. Velasco, 1995a.) [Todas las referencias indican la fecha original de publicación de la obra y las partes consultadas en las ediciones citadas en la bibliografía.]

${ }^{3} \mathrm{O}$ análogas a las metodologías que se aplican en las ciencias naturales, en el sentido de la nota anterior.
} 
Héctor Salas Harms

\section{La cientificidad de los campos del conocimiento}

El estudio de la historia de la ciencia natural permite a Thomas Kuhn (Cf. 1962: prefacio y caps. I y II) observar que el desarrollo de los campos del conocimiento o sus especialidades eventualmente llega a un punto en el que las diferentes comunidades científicas, que hasta entonces habían trabajado cada una desde los lineamientos y la metodología planteados por alguna de las teorías rivales vigentes en ese campo o especialidad, alcanzan un consenso en torno a una de ellas, reconociéndola como su primer paradigma.

La transición a la etapa paradigmática de ciencia normal —en la que la teoría universalmente aceptada 'proporciona a toda la comunidad científica los fundamentos para su práctica' desde un 'consenso firme en investigación'- puede ser más o menos extensa, pero siempre es 'extraordinariamente ardua;' y durante ella ese campo del conocimiento abandona la etapa pre-paradigmática, caracterizada por numerosas controversias sobre enfoques y métodos básicos, así como por desacuerdos abiertos relacionados con los aspectos fundamentales de la disciplina, tal como habían estado representados en las diferentes teorías alternativas incompatibles sobre un fenómeno específico simultáneamente coexistentes (Kuhn, 1962: 15).

Pero la trascendencia de tal paso radica para Kuhn en que el mismo representa un cambio cualitativo crucial en el curso de evolución de una disciplina porque "la adquisición de un paradigma y del tipo [...] de investigación que éste permite es un signo de madurez en el desarrollo de cualquier campo científico." Además, añade que "es difícil encontrar otro criterio que tan claramente proclame a un campo como ciencia" que el obtener "un paradigma que muestre ser capaz de guiar la investigación de todo el grupo" (Kuhn, 1962: 11, 22).

Desde esta perspectiva, un campo del conocimiento alcanza la madurez, pudiendo ser calificado como ciencia, cuando una de las teorías incompatibles existentes - que con su cosmovisión, leyes experimentales y métodos de investigación ofrece una alternativa para dar razón de los fenómenos objeto de su estudio- es desarrollada hasta un punto tal que su propuesta resulta convincente para todos los practicantes de la disciplina, unificando los puntos de vista hasta entonces divergentes.

$\mathrm{Al}$ respecto, Kuhn señala los periodos en que ha ocurrido esta transición en diversas ciencias o en sus especialidades. Así, desde la antigüedad se estableció en la astronomía su primer paradigma. Entre la Edad Media y el siglo XVIII, vivieron tal momento en su evolución algunas áreas de la física, tales como la dinámica, la óptica y la electricidad, en este mismo orden - y la mayor parte del resto de ellas lo tuvieron en 
Reflexiones sobre la presencia de regularidades en los fenómenos sociales

y sobre la posibilidad de investigarlas desde un monismo metodológico

el XIX. En la química tales eventos comenzaron a tener lugar a partir del siglo XVII, y las distintas partes de la biología 'cruzaron la frontera entre lo que el historiador podría llamar su prehistoria como ciencia y su historia propiamente dicha' durante los siglos XVIII y XIX. ${ }^{4}$

En esta cronología se perfila un patrón que sugiere que el orden en el que las ciencias naturales e incluso varias de sus especialidades han alcanzado su madurez está en función del creciente grado de complejidad que caracteriza a los fenómenos sobre los que trabaja cada uno de esos campos del conocimiento, a la correspondiente decreciente claridad con que se delinean y se revelan al observador esos eventos - donde él puede discernir sus regularidades - y a la consecuente progresiva dificultad para investigar y establecer empíricamente tales leyes de la naturaleza y las teorías que las describan y expliquen.

Así, el esquema anterior abarca desde las regularidades más obvias y sencillas de observar, como lo son las de la mecánica celeste hasta las de la biología, que incorpora la vida a las dimensiones físico-químicas de sus objetos de estudio. Claramente, la tarea de las disciplinas sociales, que involucra la exploración de la conducta humana en su manifestación tanto individual como grupal -e incluso asociando a ella los aspectos abstractos de elementos institucionales de distintos tipos y en diversos contextos-, muestra el mayor grado de complejidad entre los campos del conocimiento.

Sin embargo, el análisis histórico revela que los campos y especialidades que se abocan al estudio de la naturaleza uno a uno han encontrado el camino para cruzar la línea que separa el presentar una opinión personal calificada como el resultado de una investigación y la generación de conocimiento cierto y válido para todos, estableciendo desde metodologías rigurosas hechos objetivos y resultados replicables. Pero tan sólo el plantearse la necesidad de encontrar la forma de demarcar entre conocimiento y opinión encierra el compromiso de una comunidad de investigación con respecto de buscar superar la subjetividad.

\section{Las regularidades que exhiben la naturaleza y la naturaleza humana}

La concepción de un universo racional regido por leyes invariantes de aplicación general y que, por lo tanto, resulta cognoscible y predecible, es una herencia para la

${ }^{4}$ Cf. Kuhn, 1962: 20-2. El autor concluye esta síntesis señalando que 'en partes de las disciplinas sociales tales transiciones pueden muy bien estar ocurriendo ahora.' (Cf. Kuhn, 1962:21.) En sí, su investigación formal misma inició en fechas relativamente recientes, dado el alto nivel de complejidad de los fenómenos que estudian. 
actual cultura occidental por parte de la ciencia newtoniana y la filosofía empirista de la segunda mitad del siglo XVII y la primera mitad del siglo XVIII. Desde esta cosmovisión, los fenómenos del mundo y del hombre son susceptibles de ser estudiados y dilucidados, pudiendo discernir las regularidades con las que se manifiestan los eventos y señalar sus causas.

El supuesto, inferido de la experiencia ordinaria, de que existe tal uniformidad en el curso de la naturaleza, la cual se produce porque es constante el curso de cada uno de los distintos fenómenos que la componen, conduce a las ciencias naturales a ocuparse principalmente de lo generalizable a partir de lo singular. En estos términos, y más allá de la descripción de eventos naturales individuales e inconexos, la ciencia busca explicarlos investigando los patrones de recurrencia que exhiben para proponer leyes de aplicación universal que representen tales patrones, así como teorías descriptivas y explicativas de éstas. ${ }^{5}$

En uno de sus sentidos, explicar un fenómeno tanto de la naturaleza como de la naturaleza humana, con relación al cual se ha determinado por observación y experimentación que el mismo ocurre con una regularidad perceptible, es asociar a él las causas que lo producen, estableciendo una ley que vincule necesariamente el efecto particular observado con cada una de las causas del mismo que se han discernido; y proponiendo una teoría que dé cuenta de cómo y por qué sucede dicho evento. ${ }^{6}$ Asimismo, al extrapolarse la experiencia pasada, puede predecirse su ocurrencia al observarse o inducirse la presencia de esas causas; a su vez, este esquema permite la verificación de leyes y teorías, siempre que pueda establecerse la correspondencia

\footnotetext{
${ }^{5}$ De hecho, Popper (cf. 1957:59-61) afirma que "nacemos con expectativas anteriores a toda experiencia observacional como la de encontrar regularidades, que corresponde muy de cerca a la ley de causalidad que Kant consideraba como parte de nuestro equipo mental natural;' 'con una propensión innata o necesidad de buscar regularidades e imponer al mundo nuestras conjeturas, como leyes de la naturaleza.'

${ }^{6}$ Evidentemente, explicar fenómenos en términos de pretender determinar por qué propiedades ocultas de los objetos de estudio ocurren los efectos observados queda fuera de lo perceptible en toda experiencia de ellos. Por tanto, desde esta concepción, ideas como 'buscar explicaciones' o 'explorar una causalidad' se refieren tan sólo a encontrar las variables explicativas empíricas de un hecho y no a proponer 'respuestas' especulativas postulando elementos, propiedades y variables metafísicos, aspirando a dar cuenta de lo que ocurre al interior de una 'caja negra.' Así, una regularidad es entendida sólo como la uniformidad con la que eventos de cierto tipo son seguidos por otros de otro tipo, en términos de una conjunción constante entre causas y efectos propuesta ya por Hume (cf. 1739-40:lib. I, parte III, §§ I-VIII); o bien, como que "cierto hecho ocurre invariablemente siempre que estén presentes ciertas circunstancias, y no ocurre cuando están ausentes [...]" (Kolakowski, 1966:315). Asimismo, cf. Duhem (1904-5:parte I, cap. I), Hacking (1983:cap. 3) y Van Fraassen (1980:cap. 2, § 4.)
} 
Reflexiones sobre la presencia de regularidades en los fenómenos sociales

y sobre la posibilidad de investigarlas desde un monismo metodológico

entre las características de una predicción derivada de ellas, tal como han sido plasmadas en una hipótesis, y las del evento que finalmente se manifiesta. ${ }^{?}$

Lo anterior implica para la investigación científica la posibilidad de culminar proyectos generando, como hechos establecidos, resultados replicables por otros científicos, quienes partiendo de las mismas condiciones iniciales de un estudio original habrán de encontrar sus mismas consecuencias. Claramente, una expectativa semejante sugiere confiabilidad y objetividad en el conocimiento así producido, derivadas de enfocarse sobre hechos.

\section{La cientificidad de las teorías y de las proposiciones}

Planteándose la pregunta, no de 'cuándo es verdadera o aceptable una teoría,' sino de 'cuándo debe ser considerada científica una teoría' o de si 'hay un criterio para determinar el carácter o el status científico de una teoría'; Karl Popper (cf. 1957:43) señala su propósito como el de "distinguir entre la ciencia y la seudo ciencia, sabiendo muy bien que la ciencia a menudo se equivoca y que la seudo ciencia a veces da con la verdad." $"$

Para ello parte del criterio de cientificidad, o criterio de demarcación entre ciencia y no ciencia, del positivismo lógico de principios del siglo pasado, desde el cual un enunciado o una teoría son científicos si puede verificarse la representación de un hecho que buscan hacer contra la evidencia empírica que éste manifieste; es decir, si puede establecerse la verdad o falsedad de lo que afirman mediante su contrastación con la experiencia, lo cual permite fundamentar el conocimiento, determinándolo como cierto y válido para todos.

Sin embargo, Popper observa que carece de justificación lógica la pretensión de verificar una generalización a partir de someter a prueba una hipótesis que predice la ocurrencia de un evento dado, puesto que una serie finita de observaciones, como la que es posible llevar a cabo al buscar corroborarla repetidamente, no puede establecer con certeza la verdad de enunciados universales tales como leyes y teorías que están destinadas a abarcar un número infinito de casos. ${ }^{9}$ Así, cualquier muestra, por grande

\footnotetext{
${ }^{7}$ La ciencia es concebida, de acuerdo con la visión positivista, como 'el intento de codificar y anticipar experiencia,' y de desarrollar 'teorías y leyes para correlacionar, explicar y predecir datos observacionales,' con un énfasis en verificar o falsar esas teorías y leyes. (Cf. Laudan, 1990:2-3. Asimismo, cf. Popper, 1956:§§ 4-5 y Quine, 1953:79).

${ }^{8}$ Las cursivas son del autor.

${ }^{9}$ También cf. Ayer (1936:44), Lakatos (1970:92 y 94), Hempel (1950:119) y Schlick (1932-33:98.)
} 
que sea su magnitud, tiene un tamaño de cero cuando intenta representarse con ella a un universo infinito.

Por tanto, propone la falsación para reemplazar al criterio de verificación, resaltando la asimetría lógica de que mientras que un ejemplo en contrario puede refutar formalmente a una ley, ningún número de observaciones puede verificarla formalmente. Así, teorías y leyes permanecerán inevitablemente como conjeturas no verificables y de las cuales quizá nunca lleguemos a saber si son o no verdaderas, ${ }^{10}$ pero que pueden ser refutadas por un suceso que entre en contradicción con una predicción derivada de las mismas. Entonces, el criterio de cientificidad de leyes y teorías consiste en que a partir de ellas pueda realizarse una predicción susceptible de ser falsada por un evento. Consecuentemente, Popper afirma:

El criterio para establecer el status científico de una teoría es su refutabilidad [... E]s fácil obtener confirmaciones o verificaciones para casi cualquier teoría, si son confirmaciones lo que buscamos [... Sin embargo,] toda 'buena' teoría científica implica una prohibición: prohíbe que sucedan ciertas cosas. [...] Una teoría que no es refutable por ningún suceso concebible no es científica [...] Toda genuina prueba de una teoría es un intento por desmentirla, por refutarla. ${ }^{11}$

Al respecto, Popper (cf. op. cit., pp. 44-46) compara la situación de las ciencias naturales con la de las disciplinas sociales, ${ }^{12}$ señalando con relación a éstas últimas "que el elemento más característico de esa situación era la incesante corriente de confirmaciones y observaciones que 'verificaban' las teorías en cuestión"', corrían "el riesgo implicado en una predicción." Ese riesgo consiste en que cuando "la observación muestra que el efecto predicho está claramente ausente, entonces la

${ }^{10}$ Alfred Ayer (op. cit., p. 45), representante del positivismo lógico, coincide en que "ninguna proposición, fuera de las tautologías, tiene la posibilidad de ser algo más que una hipótesis probable." Asimismo, Moritz Schlick (op. cit., p. 98), fundador del Círculo de Viena y del positivismo lógico, a principios del siglo XX, señala que "las proposiciones de la ciencia que poseen mayor certeza son siempre consideradas como hipótesis constantemente abiertas a mayor precisión y perfeccionamiento." (Asimismo, cf. Popper, 1957:63.)

${ }^{11}$ [Las comillas son del autor.] Cf. Popper, 1957:47.

${ }^{12}$ Específicamente, considera la física de Einstein, por una parte; por otra, la teoría de la historia de Marx, el psicoanálisis de Freud y la psicología del individuo de Alfred Adler.

${ }^{13}$ [Las comillas son del autor.] Y continúa: “este aspecto era constantemente destacado por sus adherentes [...] Era precisamente este hecho -que siempre se adecuaban a los hechos, que siempre eran confirmadas- el que a los ojos de sus admiradores constituía el argumento más fuerte a favor de esas teorías. Comencé a sospechar que esa fuerza aparente era, en realidad, su debilidad.” (Ibid., pp. 45-6.) 
Reflexiones sobre la presencia de regularidades en los fenómenos sociales

y sobre la posibilidad de investigarlas desde un monismo metodológico

teoría queda simplemente refutada. La teoría es incompatible con ciertos resultados posibles de la observación."14

\section{Las características deseables de las teorías}

En este contexto, Kuhn (cf. 1977:321-2) enumera algunas características pertinentes de una 'buena teoría científica' consideradas usualmente. Una teoría debe ser precisa en tanto que sus predicciones o consecuencias deducibles de ella deben concordar con los hechos; debe ser consistente internamente y con otras teorías; debe tener un ámbito amplio de aplicación; debe ser simple, dando orden a fenómenos que en su ausencia estarían aislados en lo individual y que permanecerían confusos en su conjunto; además, debe revelar nuevos fenómenos aparte de explicar los ya conocidos. Asimismo, afirma que, entre todas ellas, la que muestra ser casi siempre la cualidad decisiva para preferir a una teoría sobre otra es la precisión; aunque ésta debe incluir la concordancia tanto cuantitativa como cualitativa entre las consecuencias que se derivan de ella y las observaciones y experimentos existentes, crecientemente ha llegado a denotar básicamente concordancia cuantitativa.

Claramente, las características anteriores se asocian actualmente con las teorías de las ciencias naturales. Sin embargo, Kuhn subraya que antes de principios de los tiempos modernos la precisión era un criterio aplicable solamente en astronomía, pues “[...] en ningún otro lado era esperada ni buscada." Añade que "durante el siglo

\footnotetext{
${ }^{14}$ [Las cursivas son del autor.] Posteriormente, Kuhn (cf. op. cit., caps. II, XII y VIII) pone en duda este esquema de falsación mostrando que esos planteamientos no corresponden al quehacer científico, tal como lo registra la historia de la ciencia; asimismo, argumenta que las teorías significativas nunca han explicado todos los hechos, así que si esto hubiera sido motivo para su rechazo ninguna se habría implantado. Específicamente, señala que "ningún proceso revelado hasta ahora por el estudio histórico del desarrollo científico semeja en forma alguna el estereotipo metodológico de falsación [de un paradigma] por comparación directa con la naturaleza." (Ibid., p. 77.)

Adicionalmente a este señalamiento, Ayer (cf. op. cit., pp.45-46) afirma que es tan poco posible verificar como refutar concluyentemente una hipótesis, pues al observar un hecho que la falsara, nunca existiría la certeza de que han permanecido invariables todas las condiciones relevantes, y que en realidad la hipótesis no hubiera sido invalidada. De la misma manera, Lakatos (op. cit., pp.100-101) considera que las teorías deben ser "interpretadas normalmente como conteniendo una cláusula ceteris paribus"; pero no obstante, dado un inevitable falibilismo en toda investigación, nunca podrá establecerse si ha sido refutada una hipótesis "junto con esta cláusula." [Las cursivas son del autor.]

Por último, Lakatos (op. cit., pp. 104-38), respondiendo a estos planteamientos, afirma, por una parte, que Kuhn ha cuestionado realmente una interpretación ingenua del falsacionismo y delinea un falsacionismo metodológico sofisticado que considera los anteriores señalamientos; por otra parte, abandonando el justificacionismo de Popper y del Círculo de Viena, busca devolver la racionalidad y criterio de demarcación a la ciencia desde una forma de convencionalismo. Así, en esos términos, es científica una serie de teorías en un programa de investigación si cada nueva teoría falsable conduce al progreso teórico y empírico.
} 
XVII [...] el criterio de concordancia numérica se extendió a la mecánica; a fines del siglo XVIII y principios del XIX, a la química y a otras [de las áreas de la física] tales como la electricidad y el calor; y en [el siglo XX] a muchas partes de la biología."15

Por último, Kuhn (1962:65) destaca el papel de la precisión, como una característica de las teorías en las revoluciones científicas: ${ }^{16}$

La ciencia normal ${ }^{17}$ conduce a un detalle de la información y a una precisión en la correspondencia de la teoría con la observación [... que] tienen un valor que trasciende su generalmente no muy alto interés intrínseco. [Porque] la novedad ordinariamente surge sólo para quien, sabiendo con precisión lo que debería esperar, es capaz de reconocer que algo está mal. La anomalía ${ }^{18}$ aparece sólo contra el fondo que proporciona el paradigma. Mientras más preciso y de mayor alcance es el paradigma, es más sensible como indicador de una anomalía, y por lo tanto de una ocasión para el cambio de paradigma. ${ }^{19}$

Ciertamente, las anomalías que surgen en la contrastación de teorías como una brecha entre los términos de una predicción basada en una de sus leyes y las características del hecho que se manifiesta pueden observarse sólo en teorías altamente desarrolladas en campos que han llegado a su madurez, lo cual muestra el nivel de precisión en la descripción y explicación de sus eventos que han podido alcanzar ahora las ciencias de la naturaleza en el curso de su evolución. Pero, sobre todo, muestra esas posibilidades en un contexto de fenómenos cuyas regularidades se delinean en muchos de ellos con una mayor claridad, permitiendo modelarlos y discernir más fácilmente sus variables, que asimismo se caracterizan por la presencia de constantes universales y de una causalidad de una índole tal que muestra una comparativa proximidad a los modelos del determinismo.

${ }^{15}$ Ibid., p. 335.

${ }^{16}$ El esquema de las revoluciones científicas sustituye a la propuesta de Popper de la falsación de teorías.

${ }^{17}$ Kuhn llama ciencia normal a la actividad científica realizada desde los supuestos y metodología contenidos en un paradigma, mientras permanece vigente, y que se contrapone a la ciencia extraordinaria, que tiene lugar durante la transición del paradigma anterior al que lo sustituirá, como resultado de una revolución científica.

${ }^{18}$ Una anomalía consiste en una discordancia persistente entre las expectativas generadas desde un paradigma con relación a las particularidades con que se presupone que se manifestará un fenómeno y el comportamiento con el que el mismo se observa verdaderamente en la naturaleza.

${ }^{19}$ Las cursivas son del autor. 
Reflexiones sobre la presencia de regularidades en los fenómenos sociales

y sobre la posibilidad de investigarlas desde un monismo metodológico

\section{El modelo cualitativo de investigación en las disciplinas sociales}

Los fenómenos sociales, por su parte, se caracterizan indudablemente por un mayor grado de complejidad derivada de una causalidad eminentemente probabilística, ${ }^{20}$ así como por la intervención de numerosas variables interdependientes que interactúan con efectos multiplicativos y no lineales, las cuales son no sólo de difícil medición y observación, sino que incluso eluden su aislamiento y definición para fines de su estudio y, en su caso, de su manipulación. En este contexto, buscar discernir en tales hechos regularidades que se perfilan naturalmente en forma difusa y no determinística reviste un alto nivel de dificultad en la mayor parte de los campos del conocimiento relacionados y de sus especialidades.

Consecuentemente, las teorías vigentes sobre los fenómenos humanos corresponden a las particularidades de este medio y no contemplan una expectativa de precisión ni otras de las características de las teorías sobre los fenómenos naturales. Así, partiéndose de la posibilidad de hacer predicciones meramente en términos probabilísticos -y solamente cuando hubiera podido modelarse cuantitativamente un evento-,${ }^{21}$ no hay un criterio desde el cual pueda elegirse alguna entre las distintas teorías descriptivas y explicativas de un mismo hecho que son coexistentes en el tiempo subsistiendo inevitablemente así un pluralismo teórico, aun en las áreas cuantitativas de las disciplinas sociales.

Esta situación ha llevado a cierto número de comunidades en la esfera académica de estas disciplinas a la conclusión de que los distintos aspectos de la investigación de los fenómenos sociales deben caracterizarse de una manera diferente a los de la naturaleza en términos de su 'lenguaje, estructura, objetivos, metodologías y criterios

\footnotetext{
${ }^{20}$ Sobre este concepto, vid. infra, nota 23.

${ }^{21}$ Se visualiza a un valor que predice un modelo cuantitativo como la media de una distribución normal de probabilidad que permite determinar distintos rangos dentro de cada uno de los cuales podrá esperar observarse con una probabilidad dada el verdadero valor que mostrará cierta variable al ocurrir un fenómeno. Por ejemplo, en la relación funcional inversa entre precios y cantidades, se espera que a una reducción en precios corresponda un aumento en el volumen de ventas; sin embargo, y suponiendo una función de demanda con una elasticidad unitaria en ese punto con respecto a precios, a una rebaja quizá de un $20 \%$ no se espera que siga un aumento en ventas sino de alrededor del $20 \%$. Toda vez que ésta y cualquier otra función son estimadas cuantitativamente a partir de observaciones que muestran desviaciones contra los valores que predice la curva, las bandas de confianza estadística de $95 \%$ y $99 \%$ para una predicción a partir de la misma estarán delimitadas aproximadamente dentro de dos y tres errores estándar, respectivamente, a ambos lados de la media de la distribución normal de probabilidad correspondiente, la cual es representada por la curva misma. Pero, sobre todo, cabe recordar que los extremos de esta curva son asintóticos sobre el eje horizontal; es decir, no es determinable un rango específico dentro del cual podrá encontrarse el 100\% de probabilidad para una predicción.
} 
de validez', ${ }^{22}$ rechazándose así la tesis de aplicar un solo modelo de cientificidad y racionalidad en todos los campos del conocimiento. Esta postura, por tanto, niega implícita o explícitamente la existencia de regularidades en la naturaleza humana, sosteniendo que sólo en la naturaleza pueden discernirse sus regularidades y establecerse sus leyes; en el extremo, afirma que no hay hechos sociales que puedan establecerse como ciertos y válidos para todos.

Desde esta concepción, la investigación se aboca a la realización de estudios de caso exhaustivos de índole descriptiva y con conclusiones casuísticas que no pretenden ser generalizables -derivadas de la comprensión e interpretación de las características de los fenómenos que exhiben los objetos de observación- a partir de elaborados marcos conceptuales y en el contexto de complejos modelos analíticos; estas conclusiones se basan en un criterio personal resultante de una larga formación académica y de una amplia experiencia profesional. Esta línea de pensamiento acepta tácitamente como cierto e inevitable que dos investigadores que trabajasen sobre un mismo objeto de estudio y en un mismo momento, con los mismos objetivos y siguiendo el mismo método cualitativo, no necesariamente llegasen a las mismas conclusiones.

Claramente, en una etapa pre-científica o 'clínica' en el proceso de generación del conocimiento, los estudios de caso y los diagnósticos constituyen valioso material de gran riqueza, a través de cuyo análisis pueden eventualmente discernirse a nivel hipotético las regularidades que habrán de establecerse con rigor metodológico en una etapa posterior. Inclusive, desde tales diagnósticos puede factiblemente pasarse a una etapa propositiva, generándose alternativas de solución a una problemática así determinada. En ambos casos, es la opinión personal calificada lo que se requiere.

De hecho, los modelos de racionalidad sobre la toma de decisiones, que es prerrequisito para proponer una opinión profesional, al incorporar valoraciones sobre una situación y sus causas, sobre las posibles alternativas de solución y sus resultados probables, así como sobre los criterios por elegir para tomar una decisión, 'incorporan juicios o evaluaciones personales", y "diferentes personas pueden fácilmente llegar a diferentes conclusiones cuando aplican [un mismo criterio para toma de decisiones] en un caso dado." 23

\footnotetext{
${ }^{22}$ Análogamente, en la filosofía de las disciplinas sociales "[...] han predominado dos tradiciones opuestas: la empirista, que incluye la positivista, y la tradición hermenéutica." (Cf. Velasco, 1995b:53.) "Desde la perspectiva hermenéutica, las ciencias de la cultura buscan comprender e interpretar los textos y acciones humanas, más que explicar y predecir hechos, como ocurriría en las ciencias naturales." (Ibid., p. 60.)

${ }^{23}$ Cf. Bergström, 1980:9-10.
} 
Reflexiones sobre la presencia de regularidades en los fenómenos sociales

y sobre la posibilidad de investigarlas desde un monismo metodológico

\section{La objetividad en el estudio de los fenómenos de la naturaleza humana}

Sin embargo, más allá de las opiniones, la ciencia debe basarse en hechos ciertos y válidos para todos. Pero ante el alto grado de dificultad que caracteriza a la investigación de los eventos sociales resulta controvertida no la cuestión de si es deseable trazar una línea de demarcación entre conocimiento y opinión en las disciplinas sociales, sino la cuestión de si esto es posible; es decir, si es posible en un ámbito como éste ir más allá de la subjetividad para establecer hechos objetivos y discernir regularidades mediante estudios replicables.

Aquí, son pertinentes las reflexiones de León Olivé (cf. 1995:100-101) por una parte, "una creencia es objetiva porque se refiere a un hecho objetivo;" y, por otra, "para poder calificar a una creencia de auténtico conocimiento, se requiere que las razones que la apoyan sean buenas razones." Éstas "son las objetivamente suficientes para aceptar la creencia en cuestión." ${ }^{24}$ En este punto, el autor incorpora a su argumentación dos citas de Luis Villoro (1982:137-138), cuyo texto completo es ilustrativo:

'Razones objetivamente suficientes'son las que bastan para garantizar la verdad de la creencia, con independencia del juicio de quienes creen. Pero como la creencia sólo es verdadera si existe realmente el objeto o situación objetiva creídos, 'razones objetivamente suficientes' son las que garantizan que la creencia está efectivamente determinada por la realidad y no por motivos subjetivos de quienes creen. Pero entonces, 'objetivo' es aquello cuya validez no depende del punto de vista particular de una o varias personas, sino que es válido con independencia de este punto de vista, para todo sujeto de razón que lo considere. ${ }^{25}$

Ciertamente, la subjetividad está contrapuesta al conocimiento. Olivé (op. cit., p. 102) recapitula señalando que "podemos entender la objetividad como aceptabilidad racional en condiciones realmente existentes para una comunidad epistémica [...] La objetividad se refiere, pues, a la posibilidad de reconocimiento público, en una comunidad determinada, de que hay una situación de hecho." 26

En estos términos, es claro que en los fenómenos sociales hay situaciones de hecho, aunque cabe señalar que gran número de esos eventos y sus variables no son empíricos y que tales variables se definen y se miden desde metodologías establecidas

\footnotetext{
${ }^{24}$ Las cursivas son del autor.

${ }^{25}$ Las comillas son del autor.

${ }^{26}$ Las cursivas son del autor.
} 
en forma convencional y consensuada en una comunidad de esa disciplina. ${ }^{27}$ Pero entonces, en tanto los conceptos y variables de los fenómenos bajo estudio puedan ser definidos y medidos en forma no subjetiva, puede llevarse a cabo investigación cuantitativa para prueba de hipótesis con rigor metodológico y pueden establecerse sus conclusiones e implicaciones para teoría como hechos objetivos mediante trabajos replicables por otros investigadores.

Adicionalmente, desde estas metodologías pueden efectuarse análisis cuantitativos con modelos causales para explicar las regularidades que se disciernen en los fenómenos de la naturaleza humana y establecer sus determinantes como hechos ciertos y válidos para todos mediante proyectos con resultados y conclusiones replicables por investigación subsiguiente. Asimismo, a partir de estos modelos cuantitativos podrán generarse las predicciones probabilísticas pertinentes para la toma de decisiones en las organizaciones.

Ahora bien, regresando en este punto a los planteamientos de Olivé, puede observarse que sus consideraciones sobre la objetividad se aplican indistintamente a los hechos aquí señalados, los cuales tendrán 'aceptabilidad racional en condiciones realmente existentes para una comunidad epistémica', así como el 'reconocimiento público, en una comunidad determinada, de que hay una situación de hecho.'

\footnotetext{
${ }^{27}$ Son situaciones de hecho no perceptibles empíricamente, por ejemplo, en administración, un cambio de estrategia en una firma, una caída en su participación de mercado o una fusión, adquisición o quiebra de empresas; o en economía, la devaluación de una moneda, un crack bursátil o una recesión. O bien, en finanzas y econometría, son hechos no empíricos en cuanto a la primera, un aumento en la rentabilidad de un negocio, la reducción en su costo de capital o el precio de cotización de la oferta pública inicial de su acción; en relación con la segunda, un aumento en la productividad marginal del trabajo por cambios tecnológicos, una inversión de la curva de tasas de interés por cambios en expectativas o una disminución de la volatilidad de rendimientos bursátiles.

Por su parte, tampoco son empíricas las variables asociadas a estos hechos y se miden desde metodologías convencionales - al igual, desde luego, que los parámetros de sus funciones explicativas-; pero los valores que adoptan todos ellos en cada momento quedan establecidos como hechos válidos para todos. Con respecto a los ejemplos citados de finanzas, lo son variables y sus valores tales como el ROI o ROA de ese negocio al final de un periodo, el costo de cada renglón de su pasivo y de su capital y su tasa promedio ponderada global en una fecha dada o la curva de demanda inicial por su acción y el precio al que se cierra esa transacción; y en relación con los de la segunda área, lo son variables, parámetros y sus valores tales como los coeficientes de una función de producción en un lapso dado, las tasas de interés y plazos de vencimiento de deuda, así como las expectativas con respecto a aquéllas en cierto momento o la varianza de los precios diarios de acciones y, en general, numerosos casos más tales como el valor del INPC al final de una quincena y su cambio porcentual durante la misma, el saldo y el flujo de M1 o M3 durante un periodo, el tipo de cambio de apertura de cierto día, el saldo en una de las cuentas nacionales al final de un año, los márgenes de operación o neto de una compañía, o la UPA, dividendo, múltiplo P/U o precio de cierre de su acción en cierta fecha, etcétera.
} 
Reflexiones sobre la presencia de regularidades en los fenómenos sociales

y sobre la posibilidad de investigarlas desde un monismo metodológico

Es decir, que no estará sujeto a duda si hay situaciones de hecho realmente existentes con respecto a los eventos sociales, aun cuando no sean empíricamente perceptibles. Lo que puede ser controvertido son las metodologías convencionales empleadas para la definición y cuantificación de las variables relacionadas y, por lo tanto, los resultados obtenidos. Pero dado el caso de que desde diferentes supuestos y metodologías llegaran a establecerse resultados y conclusiones equivalentes, habría buenas razones para sostener los hechos así determinados, que es lo más usual en los estudios cuantitativos económico-financieros.

\section{Los hechos en los fenómenos sociales}

Mediante análisis cuantitativo aplicado a variables definidas y medidas bajo metodologías convencionales pueden establecerse como hechos objetivos, entre otros, los siguientes:

- si las tendencias de las series de tiempo de distintas variables financieras cambian en sincronía con la transición de una etapa a otra a lo largo del ciclo de vida del negocio al que pertenece una organización (cf. Gómez, 2004);

- si los costos unitarios de operación disminuyen conforme aumenta la escala misma de operación de las compañías que pertenecen a ciertos giros (cf., p. e., Merino, 1985);

- si una de las estrategias de crecimiento alternativas se asocia con un mejor desempeño financiero y de mercado de las empresas que la adoptan (cf. Rumelt, 1986);

- si las acciones muestran consistentemente diferentes niveles de riesgo en función de la industria en la que operan las firmas (cf., p. e., Ibbotson Associates, 2006a y 2006b);

- si los precios de las acciones son una serie de fluctuaciones aleatorias restringidas alrededor de sus valores intrínsecos (para el planteamiento teórico, $c f$., Cootner, 1962);

- si el riesgo total de una cartera de inversión diversificada en forma aleatoria se reduce sustancialmente al adicionar activos hasta aproximadamente 15 de ellos, y a partir de allí no puede reducirse el significativamente riesgo total ( $c f$., Evans y Archer, 1968);

- si tiene lugar una reacción oligopólica en los sectores industriales que emprenden un proceso de inversión directa en el extranjero (cf., Knickerbocker, 1973);

- si imperfecciones de mercado como la información asimétrica producen distorsiones en la asignación del crédito bancario a las micro y pequeñas empresas (cf., p. e., Chablé, 2006); o

- si los métodos Black \& Scholes y Valor económico agregado consistentemente sobrevalúan y subvalúan, respectivamente, las valuaciones de acciones producidas por Flujos de efectivo descontados (cf. Saavedra, 2002). 
Por supuesto, a esta lista pueden añadirse muchos casos más. Claramente, en las disciplinas sociales es posible producir resultados que queden establecidos como hechos ciertos y válidos para todos los practicantes en una de ellas mediante estudios replicables por otros investigadores, quienes podrán someter a corroboración esas conclusiones; dichos estudios se llevan a cabo desde metodologías aceptadas convencionalmente en acuerdos consensuados al interior de las comunidades de investigación de esa disciplina. No obstante a todo énfasis que pueda ponerse en lo anterior, ciertamente podrán surgir cuestionamientos sobre la naturaleza, elementos y supuestos de la teoría en la que se enmarcan cada uno de esos proyectos, así como sobre los nuevos supuestos y propuestas adoptadas para solucionar los problemas metodológicos específicos encontrados durante los trabajos correspondientes.

Sin embargo, es esto precisamente lo que ha de enfrentarse y resolverse en todo tipo de investigación. Larry Laudan ( $c f .1990: 95-7$ y ss.), filósofo pragmatista contemporáneo, en referencia a la investigación en ciencias naturales, afirma que "las reglas de la metodología deben ser convenciones," toda vez que "no son empíricas ni evidentes por sí mismas." ${ }^{28}$ Asimismo, y ya que las teorías no pueden verificarse ni falsarse formalmente, ${ }^{29}$ es desde decisiones metodológicas consensuadas en las comunidades de investigación, a partir de la evidencia empírica acumulada por la investigación previa, que se lleva a cabo la elección de una entre ellas; el criterio para establecer su validez es referido a consideraciones heurísticas y pragmáticas. Al respecto, Laudan (ibid., pp. 102-3) añade, en relación con todos los elementos y supuestos de la metodología para contrastar una teoría que son sujetos a cuestionamiento, que:

La ciencia es una herramienta altamente efectiva y exitosa para generar expectativas sobre el mundo natural. Yo noto, [...] que la ciencia parece también ser una actividad gobernada por reglas. Me digo: 'algo debe ser responsable por el impactante éxito de las teorías científicas.' [... Por lo tanto,] a menos que las reglas del método científico reflejen algo en 'materia de hechos,' la investigación científica no sería en lo absoluto tan exitosa como lo es [... en permitirnos] controlar, predecir y manipular nuestro ambiente. ${ }^{30}$

Y queda abierto a consideración en qué medida es aplicable esto a los fenómenos sociales; es decir, en qué medida ha sido posible también 'controlar, predecir y manipular nuestro ambiente' social a partir de las teorías administrativas, económicas y otras.

\footnotetext{
${ }^{28}$ Las cursivas son del autor.

${ }^{29}$ Cf. supra, pp. 6-7 y nota 16. Asimismo, sobre la infradeterminación empírica de las teorías y la justificación del convencionalismo, $c f$. Ayer (1936:45-6), Lakatos (1970), Duhem (1904-5, parte II, cap. VI, $\S \S$ II-III y VIII-X) y Laudan (op. cit., cap. 2.)

${ }^{30}$ Las comillas son del autor.
} 
Reflexiones sobre la presencia de regularidades en los fenómenos sociales

y sobre la posibilidad de investigarlas desde un monismo metodológico

\section{Las regularidades en los fenómenos sociales}

Por último, pueden también determinarse las funciones explicativas de las regularidades observadas en los fenómenos económico-financieros mediante análisis cuantitativo con metodologías convencionales en proyectos replicables. ${ }^{31}$ Las relaciones causales entre una variable y sus determinantes han sido investigadas en ambas disciplinas durante décadas. Funciones como las de tasas de interés, inflación, inversión e inversión extranjera directa o tipo de cambio; o bien, de captación y demanda por crédito bancario, beneficios totales y costo de capital o en valuación de acciones y activos, y muchas otras, están firmemente establecidas, forman parte de su acervo teórico y empírico. Estos trabajos cuantitativos ciertamente parten del carácter probabilístico de los fenómenos mismos y sus consecuentes limitaciones en toda pretensión de predecir la conducta humana y los eventos sociales.

Sin embargo, en estas disciplinas, esas metodologías limitan la exploración de causas y efectos a fenómenos cuyas variables son cuantificables. Pero, sobre todo, cuando busca determinarse una causalidad en relación con acciones humanas — tales como consumir, invertir o adoptar una estrategia- hay un sesgo inherente en esquemas de investigación que llevan a cabo análisis ex post facto -o después de que el hecho ocurrió y, por ello, con muestras que contienen solamente los casos en los cuales se llevó a cabo la acción en cuestión- para buscar establecer relaciones causales que naturalmente son ex ante facto. Es decir, las variables que son determinantes de una decisión para emprender una acción tienen un papel definitivo en un momento anterior a la acción.

Por ejemplo, la estimación por análisis cuantitativo de una función de inversión extranjera directa a partir de muestras que incluyen exclusivamente países receptores de IED no arroja coeficientes significativos para el riesgo como variable explicativa, pues el diseño de investigación ex post presupone menor inversión a mayor riesgo. Pero el análisis ex ante, con muestras tanto de países en los que se invirtió como en los que no fue así, no presupone que cuando un inversionista percibe que el riesgo es alto invertirá una cantidad menor, sino que la inversión simplemente no ocurrirá. ${ }^{32}$ En el primer caso, habría intentado encontrarse significación de coeficientes de regresión con datos cruzados de una muestra de países que recibieron IED, mientras que en el segundo puede encontrarse una diferencia significativa entre el riesgo medio de los países receptores y el de los no receptores de IED.

\footnotetext{
${ }^{31}$ En otras de las disciplinas sociales se realizan también trabajos de este tipo.

${ }^{32}$ Cf. Kobrin, 1976:30-32 y Stevens 1974:71.
} 
El enfoque ex post permite explorar los determinantes de decisiones tales como aportar capital de riesgo a proyectos de negocios, adoptar cierto estilo de liderazgo, adecuar la estructura organizacional o cambiar las estrategias de crecimiento o de competencia, entre muchas más. El análisis ex ante permite estudiar aun eventos con variables no cuantificables, examinándose en ambas muestras (la de casos en los que se tomó tal decisión y la de casos contrarios) las variables discriminantes de la ocurrencia de tales fenómenos. Así, puede establecerse en forma objetiva, cierta y válida para todo investigador - a partir de muestras representativas y en proyectos replicables- que la presencia o ausencia de ciertos elementos causales define el que se realice o no cierto hecho social.

De esta manera pueden establecerse los determinantes de ventajas competitivas en el nivel mundial que han desarrollado diversos sectores industriales y que han podido mantenerlas durante periodos sumamente largos; ${ }^{33}$ los de ambientes económicos, políticos, legales, científicos, culturales, etc., que son propicios para nutrir el surgimiento de nuevos negocios en industrias de tecnologías de punta; los de ambientes industriales, laborales, fiscales, culturales, entre otros, que son favorables para el desarrollo de empresas altamente innovadoras; o muchos otros. O, bien, pueden definirse los factores discriminantes de la supervivencia del 5\% de nuevas microempresas más allá de los primeros dos años desde su creación y los que caracterizan al 95\% que no lo hacen; los de fusiones y adquisiciones, dentro del mismo giro o hacia fuera del mismo, que fracasan y los de aquellas que tienen éxito; o los de acciones que permanecen altamente sobrevaluadas durante largos periodos, entre muchos casos más.

\section{Conclusiones}

En términos de las consideraciones anteriores, las disciplinas sociales no habrían alcanzado un estatus de cientificidad desde los criterios de Kuhn, Popper y Lakatos, por lo que aún se encontrarían en una etapa pre-paradigmática sin consenso en las comunidades de investigación aun sobre la naturaleza de los fenómenos sociales y subsistiendo en ellas un pluralismo teórico, con teorías que no serían científicas ni buscan precisión en la descripción y explicación de estos últimos. De acuerdo con tales planteamientos, cientificidad y precisión caracterizarían hoy sólo a las ciencias naturales y a cada una de ellas a partir de un momento histórico cada vez más reciente en función de la creciente complejidad de los eventos que estudia.

La investigación de los hechos sociales para discernir regularidades que ocurren en forma difusa y desde una causalidad probabilística, en los que intervienen numerosas

\footnotetext{
${ }^{33}$ Cf. Porter (1998).
} 
Reflexiones sobre la presencia de regularidades en los fenómenos sociales

y sobre la posibilidad de investigarlas desde un monismo metodológico

variables que interactúan entre sí con efectos multiplicativos y no lineales y que son de difícil definición, medición y observación, genera gran controversia. Así, diversas comunidades académicas consideran inviable su explicación y predicción conforme a los objetivos y metodologías del modelo naturalista de cientificidad y racionalidad; desde la concepción de un dualismo metodológico para ciencias naturales y disciplinas sociales - paralelo al que se presenta en la filosofía de la ciencia- proponen que la investigación debe enfocarse en estas últimas sólo a la comprensión e interpretación de los eventos sociales para describirlos de manera casuística y no generalizable a través de métodos que conducen a resultados subjetivos.

Por otra parte, la investigación basada en los planteamientos de un monismo metodológico busca establecer las regularidades percibidas en los fenómenos de la naturaleza humana, al igual que distintos eventos sociales, como hechos ciertos, válidos y corroborables por todo investigador mediante metodologías cuantitativas de carácter convencional, desde las cuales pueden también definirse y medirse sus variables de manera consensuada; lo anterior permite sugerir confiabilidad y objetividad en las conclusiones generadas de este modo y superar el esquema de emitir una opinión personal calificada como el resultado de una investigación.

En esta línea - y con referencia a administración y finanzas, así como a economíase han mostrado:

a) Eventos no empíricos que constituyen situaciones de hecho ciertas y válidas para todos, cuyas variables igualmente no empíricas se definen y se miden convencionalmente desde metodologías consensuadas, cuyos valores en función de éstas quedan establecidos también como hechos objetivos en todo momento en que las mismas los adoptan.

b) Eventos no empíricos con variables no empíricas de medición convencional, en los cuales las relaciones constantes de índole causal o no causal entre ellas pueden también establecerse como hechos ciertos y válidos para todo investigador mediante pruebas de hipótesis con muestras representativas y metodologías rigurosas que permiten generar resultados y conclusiones generalizables y replicables por investigación subsiguiente.

c) Fenómenos no empíricos con variables no empíricas de medición consensuada, en los que buscan discernirse regularidades asociándose a ellos sus causas mediante funciones explicativas pudiendo establecerse sus determinantes como hechos ciertos, válidos y corroborables por todo investigador en proyectos cuantitativos con rigor metodológico y modelos causales para prueba de hipótesis, los cuales permiten derivar resultados y conclusiones generalizables y replicables. A su vez, y a partir de las estimaciones que constituyen esos mo- 
delos cuantitativos, pueden generarse predicciones probabilísticas pertinentes para la toma de decisiones en las organizaciones.

Por último, se muestra que la exploración de causas y efectos puede extenderse incluso a fenómenos cuyas variables no son cuantificables, superándose también el sesgo inherente en esquemas de investigación en que se realizan análisis con datos ex post facto cuando busca determinarse una causalidad en relación con acciones humanas. Así, toda vez que en la decisión de emprender una acción las relaciones causales con sus determinantes se dan en un momento anterior a la acción, los diseños de investigación ex ante permiten establecer en forma objetiva, cierta y válida para todo investigador - a partir de muestras representativas y en proyectos replicables- que la presencia o ausencia de ciertos elementos causales define el que se realice o no un hecho social; este esquema permite también definir los factores discriminantes de la ocurrencia de hechos sociales ajenos a decisiones y control humanos.

\section{Bibliografía}

AYER, Alfred Jules, Language, truth and logic, Víctor Gollancz, Ltd., Londres, 1956 (1a. ed., 1936).

BERGSTRÖM, Lars, Some remarks concerning rationality in science, en Risto Hilpinen (ed.), D. Reidel Publishing Company, Londres, 1980.

CHABLÉ SANGEADO, Juan José, La importancia de la información financiera en el acceso al crédito de micro y pequeñas empresas manufactureras en Tabasco, tesis doctoral inédita, Doctorado en Ciencias de la Administración, unAM, México, 2006.

COOTNER, Paul H., "Stock prices: random versus systematic changes", en Industrial Management Review, vol. 3, núm. 2, spring 1962, pp. 24-45.

Duhem, Pierre, La théorie physique. Son obje-sa structure, Lib. Phil. J. Vrin, París, 1993. (1a. ed., 1904-05.)

EVANS, J. y S. H. ARCHER, "Diversification and the reduction of dispersion: an empirical analysis," en Journal of Finance, diciembre, 1968, pp. 761-767.

GÓMEZ ALVARADO, Luis A., Evolución de variables financieras a lo largo del ciclo de vida, tesis doctoral inédita, Doctorado en Ciencias de la Administración, UNAM, México, 2004.

HACKING, Ian, Representing and intervening, Cambridge University Press, Londres, 1983. 
Reflexiones sobre la presencia de regularidades en los fenómenos sociales

y sobre la posibilidad de investigarlas desde un monismo metodológico

HEMPEL, Carl Gustav, "Problemas y cambios en el criterio empirista de significado", en A. J. Ayer (comp.), El positivismo lógico, (tr. L. Aldama et al.), FCE, México, 1965 (Publicado originalmente en 1950).

HUME, David, Treatise of human nature, L. Selby-Bigge (ed.), Clarendon P., Oxford, 1988 (1a. ed., 1739-40).

IBBOTSON ASSOCIATES, 2006 International equity risk premia report, Ibbotson Associates, Chicago, 2006a.

, 2006 Risk premia over time report, Ibbotson Associates, Chicago, $2006 \mathrm{~b}$.

KNICKERBOCKER, F. T., Oligopolistic reaction and multinational enterprise, Harvard University Press, Boston, 1973.

KOBRIN, Stephen J., "The environmental determinants of foreign direct manufacturing investment: an ex post empirical analysis", en Journal of International Business Studies (fall / winter), 1976, pp. 29-42.

KolaKowski, Leszek, La filosofía positivista, (tr. G. Ruiz Ramón), Cátedra, Madrid, 1981 (1a. ed. 1966).

KUHN, Thomas S., The structure of scientific revolutions, University Chicago Press-Phoenix, 1964 (1a. ed., 1962).

, The essential tension, University of Chicago Press, 1977.

LAKATOS, Imre, "Falsification and the methodology of scientific research programmes", en I. Lakatos y A. Musgrave (eds.), Criticism and the growth of knowledge, Cambridge University Press, 1970.

LAUDAn, Larry, Science and Relativism, University of Chicago Press, 1990.

MERINO RENDÓn, Gregorio, Economías de escala en el sistema bancario mexicano, Tesis profesional inédita, ITAM, México, 1985.

OLIVÉ, León, “Racionalidad, objetividad y verdad”, en L. Olivé (ed.), La racionalidad epistémica, Enciclopedia Iberoamericana de Filosofía, vol. 9, Trotta-csic, Madrid, 1995, pp. 91-121.

POPPER, Karl R., "Tres concepciones sobre el conocimiento humano", en K. R. Popper, El desarrollo del conocimiento científico: Conjeturas y refutaciones, (tr. Néstor Miguez), Paidós, Buenos Aires, 1979 (1a. ed., 1956).

, "La ciencia: conjeturas y refutaciones", en K. R. Popper, El desarrollo del conocimiento científico: Conjeturas y refutaciones (tr. Néstor Miguez), Paidós, Buenos Aires, 1979 (1a. ed., 1957). 
PORTER, Michael E., Competitive advantage of nations, Simon \& Schuster Trade, Nueva York, 1998.

QUINE, Willard V. O., Desde un punto de vista lógico (tr. M. Sacristán), Ariel, Barcelona, 1962 (1a., ed 1953).

RUMELT, Richard P., Strategy, structure and economic performance, Harvard Business School Press, Boston, 1986.

SAAVEDRA GARCÍA, María Luisa, La valuación de empresas: enfoques teóricos y aplicación de los modelos Black y Scholes, Valor económico agregado y flujo de efectivo disponible en México 1991-2000, tesis doctoral inédita, Doctorado en Ciencias de la Administración, unAM, México, 2002.

SCHLICK, Moritz, "Positivismo y realismo", en A. J. Ayer (comp.), El positivismo lógico, (tr. L. Aldama, et al.), FCE, México, 1965. (Primera edición, 1932-33.)

STEvens, G. V., "The determinants of investment", en J. H. Dunning (ed.), en Economic analysis and multinational enterprise, George Allen \& Unwin, Ltd., Londres, 1974.

van fraAssen, Bas C., The scientific image, Clarendon Press, Oxford, 1980.

VELASCO GÓMEZ, Ambrosio, "Filosofía de la ciencia, hermenéutica y ciencias sociales", en Ciencia y desarrollo, vol. XXI, no. 125, nov.-dic., México, 1995a, pp. 69-81.

-, "La hermeneutización de la filosofía de la ciencia contemporánea", en Diánoia/ Anuario de filosofía, vol. XLI, México, 1995b, pp. 53-64.

VILloro, Luis, Creer, saber, conocer, Siglo XXI Editores, México, 1982. 